\title{
Deciphering the nature of ion-graphene interaction
}

\author{
Ke Zhou (10) and Zhiping Xu* \\ Applied Mechanics Laboratory, Department of Engineering Mechanics, Tsinghua University, Beijing 100084, China
}

(Received 28 January 2020; revised 28 June 2020; accepted 31 August 2020; published 1 December 2020)

\begin{abstract}
Ion-specific interactions with materials are important for the design of ion-based energy and information devices, which are studied here with a focus on alkali-earth-metal and transition-metal ions in the context of carbon nanofluidics. Density functional theory calculations show that for the alkali-earth-metal ions, the nature of interaction is ionic, with charge transfer to and from graphene determined by the change in work function. In contrast, covalent bonding is identified for transition-metal ions adsorbed on graphene, showing hybridization near the Dirac point. The ion-graphene interaction is significantly stronger than van der Waals interactions and shows much higher contrast among the ions. We find that, surprisingly, these features are robust even with the presence of water solvation, where alkali-earth-metal ion-graphene interaction remains ionic with modified charge transfer, while covalent interaction with transition-metal ions turns into ionic. These understandings imply that the ion-wall interaction can be notably tuned by the external electric field, as verified by our calculations, and opens an avenue for the development of nanofluidics where the channel width is comparable with the range of ion-wall interaction.
\end{abstract}

DOI: 10.1103/PhysRevResearch.2.042034

Charge-neutral, chemically inert surfaces such as graphene or graphite are well known to interact with many atoms or molecules via van der Waals interactions. These long-range, nonbonding interactions are not only weak in the strength but also less discernible between the absorbed species and lack of external control. Interestingly, recent studies in nanofluidics show that ions in graphene nanochannels demonstrate highly selective transport behaviors [1-6]. These unexpected findings were attributed to the contrast in the ion-wall interaction [5-7]. The high contrast in ion-wall or ion-pore interaction also indicates discernible activation energies for diffusive processes of ions, offering plenty of room for recognition and selectivity of ions [8-10]. For example, recent experiments show that nanopores decorated by crown ethers impressively promote the $\mathrm{K}^{+} / \mathrm{Na}^{+}$selectivity to more than 50 [10].

The notion of cation- $\pi$ interaction was proposed to explain the noncovalent and relatively strong $(\approx 1.0 \mathrm{eV})$ interaction between (hydrated) cations and the aromatic rings [11-15]. The cation- $\pi$ interaction stems from the interaction between the ion and induced dipole, quadruple moments of the $\pi$ system [11-13], which allows the control of molecular recognition, catalysis, and physiological processes $[11,12,16,17]$. Moreover, it leads to one order-of-magnitude difference in the energy of adsorption [11-14]. This concept is referred to explain highly selective ion transport in nanochannels through the contrast in the ion-wall interaction

\footnotetext{
*Corresponding author: xuzp@tsinghua.edu.cn

Published by the American Physical Society under the terms of the Creative Commons Attribution 4.0 International license. Further distribution of this work must maintain attribution to the author(s) and the published article's title, journal citation, and DOI.
}

that contributes to the drag force [5-7]. However, the nature of cation- $\pi$ ion-wall interaction in carbon nanofluidics has not been well elucidated. The effects of solvents are unknown, and how the interaction can be controlled through external cues has not been discussed. Exploration in this context could shed light on the design of ionic devices for mass and/or information transport, storage, and detection, offering fundamental understanding of physical processes in biology and chemistry [2,3,11,12,15-19].

In this Rapid Communication, we employed firstprinciples calculations at the level of density functional theory (DFT). The functional of strongly constrained and appropriately normed (SCAN) meta-generalized gradient approximation (meta-GGA) is used to probe the nature of ion-graphene interaction, the results of which are compared with other functionals, with or without van der Waals corrections [20-22]. A wide spectrum of cations including $\mathrm{Na}$, $\mathrm{K}, \mathrm{Ca}, \mathrm{Cu}, \mathrm{Cr}, \mathrm{Fe}$, and $\mathrm{Ni}$ were studied, covering distinct electronic structures and chemical binding behaviors. The strength of ion-wall interaction with graphene and the bonding characteristics were assessed, followed by discussion on the effects of solvent and external electric field (EEF), to address the robustness and tunability in practical applications.

To study the ion-wall interaction, we constructed a $4 \times 4 \times$ 1 supercell of graphene ( 32 carbon atoms) with a lattice constant that is relaxed to $2.46 \AA$. A single metal atom is placed on top of the graphene, at three high-symmetry sites, the center of hexagons (hexagon, $\mathrm{H}$ ), the top of carbon atoms (top, $\mathrm{T}$ ), and the bond center (bond, B). The ion-graphene system is charge neutral. The vacuum layer thickness $(H)$ in the $z$ direction is set to $15 \AA$ for bare graphene and that with ion absorption $20 \AA$ for hydrated ions, as the diameter of hydration is $\approx 5 \AA$ [23]. Spin-polarized calculations were performed using the Vienna $A b$ Initio Simulation Package (VASP) with a kinetic energy 
(a)

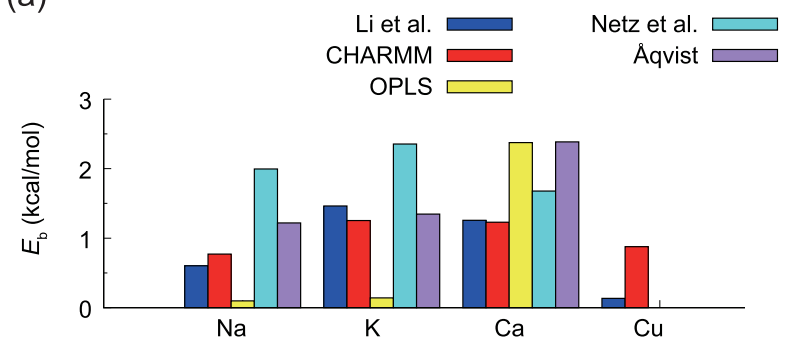

(b)

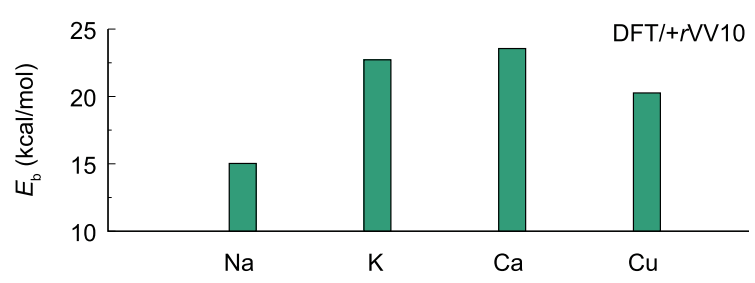

(c)

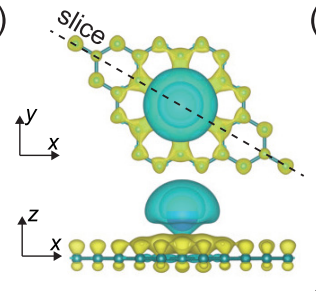

(f)

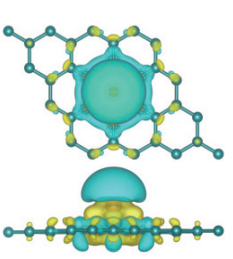

(i)

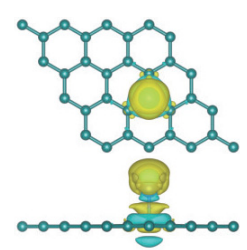

(d)

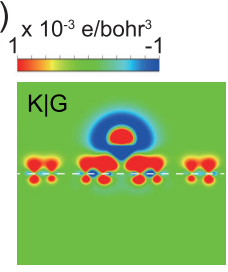

(g)

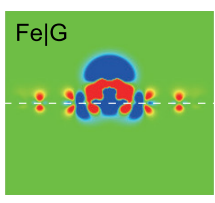

(h)
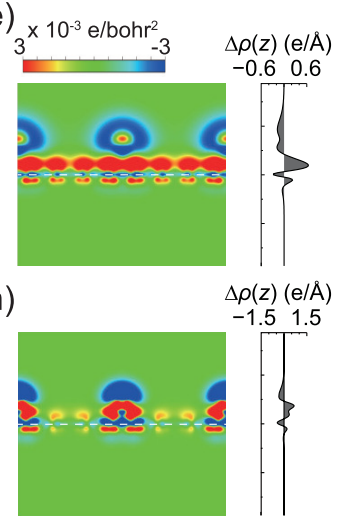

(j)

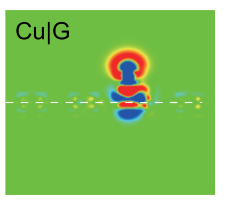

$(\mathrm{k})$

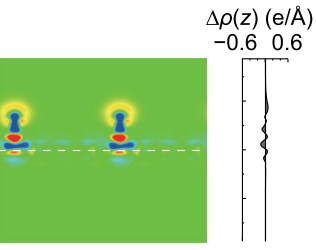

FIG. 1. [(a), (b)] Binding energies $\left(E_{\mathrm{b}}\right)$ of $\mathrm{Na}, \mathrm{K}, \mathrm{Ca}$, and $\mathrm{Cu}$ on graphene as calculated from 12-6 L-J potential functions [22] and DFT/SCAN $+r$ VV10. The heteroatomic L-J force-field parameters are computed through the Lorentz-Berthelot mixing rules [22]. [(c), (f), (i)] Differential charge densities $(\Delta \rho)$ of $\mathrm{K}|\mathrm{G}, \mathrm{Fe}| \mathrm{G}$, and $\mathrm{Cu} \mid \mathrm{G}$ composites at the energy-favorable sites ( $\mathrm{H}, \mathrm{H}$, and $\mathrm{T}$, respectively). Isosurfaces are visualized at $1.0 \times 10^{-3} \mathrm{e} / \mathrm{bohr}^{3}$ (yellow for gain and cyan for loss). [(d), (g), (j)] 2D slices of $\Delta \rho$. [(e), (h), (k)] Accumulated $\Delta \rho$ in the $x-y$ plane. The line plots on the right show the values of $\Delta \rho$ along the $z$ direction, which is averaged in the $x-y$ plane.

cutoff of $500 \mathrm{eV}[24,25]$ and dipole-moment corrections in the $z$ direction [26]. A $9 \times 9 \times 1, \Gamma$-center Monkhorst-Pack mesh grid was used for Brillouin-zone integration. The projector augmented wave (PAW) method is used for the ion-electron interaction [27], and pseudopotentials for $\mathrm{Na}(\mathrm{K}), \mathrm{Ca}, \mathrm{Cu}, \mathrm{Cr}$, $\mathrm{Fe}$, and Ni contain the $p^{6} s^{1}, p^{6} s^{2}, d^{10} s^{1}, d^{5} s^{1}, d^{6} s^{2}$, and $d^{8} s^{2}$ shells, which are treated as valence states. To include the longrange van der Waals interactions in SCAN meta-GGA that can well describe intermediate-range van der Waals interactions [20,21], the revised Vydrovvan Voorhis nonlocal correlation ( $r$ VV10) is adopted [21].

The adatoms adsorbed on graphene are ionized [28,29], and the energy of binding or adsorption for ions, $E_{\mathrm{b}}$, is calculated as $E_{\mathrm{b}}=E_{\mathrm{M} \mid \mathrm{G}}-E_{\mathrm{M}}-E_{\mathrm{G}}$. Here $E_{\mathrm{M} \mid \mathrm{G}}$ is the total energy of geometrically optimized atomic structures of the composite system and $E_{\mathrm{G}}, E_{\mathrm{M}}$ are those for isolated systems of graphene and the ion, respectively. The calculation results show that these ions can be divided into two categories according to the bonding characteristics of their interaction with graphene, which is ionic for $\mathrm{Na}, \mathrm{K}$, and $\mathrm{Ca}$, but covalent for $\mathrm{Cu}, \mathrm{Cr}, \mathrm{Ni}$, and $\mathrm{Fe}$. The results show that the value of $E_{\mathrm{b}}$ is on the order of $1.0 \mathrm{eV}$ (Table S1) [22], which is consistent with that of ions on benzene or other aromatic molecules $[11,12,14]$. In contrast, the value of $E_{\mathrm{b}}$ calculated from the 12-6 Lennard-Jones (LJ) potential functions that are parametrized in widely used force fields [22], $\approx 0.1 \mathrm{eV}$, is significantly lower (Fig. 1). The enhanced strength of adsorption, as attributed to the cation$\pi$ interaction in the literature, requires clarification of the ion-wall interaction and a more reasonable parametrization procedure in force-field development.

To understand the contrast in ion-graphene adhesion, we analyzed electronic structures of the $M \mid G$ composites.
The work functions of single atoms $\left(W_{\mathrm{M}}\right)$ was calculated, which demonstrate a linear correlation with the ionization energy (IE) [22]. The values of $W_{M}$ are compared with that of graphene $\left(W_{\mathrm{G}}=4.29 \mathrm{eV}\right)$, showing that, for alkali-earthmetal ions $\mathrm{Cr}$ and $\mathrm{Ni}\left(W_{\mathrm{M}}<W_{\mathrm{G}}\right)$, charge is transferred into graphene, in consistency with the conclusion from Bader charge analysis [22]. For transition-metal ions such as $\mathrm{Cu}$ and $\mathrm{Fe}$, we have $W_{\mathrm{M}}>W_{\mathrm{G}}$, but the ion-wall interaction has a covalent nature, and thus graphene is not an electron donor. The shift in the work function, $W_{\mathrm{M} \mid \mathrm{G}}-W_{\mathrm{G}}$, scales linearly with the electric-dipole moment, $M[22,30]$. To elucidate the bonding characteristics, the redistribution and transfer of charge densities in the real space were analyzed (Fig. 1). We define the charge-density difference as $\Delta \rho=\rho_{\mathrm{M} \mid \mathrm{G}}-\rho_{\mathrm{M}}-\rho_{\mathrm{G}}$, where $\rho_{\mathrm{M} \mid \mathrm{G}}, \rho_{\mathrm{M}}$, and $\rho_{\mathrm{G}}$ are the charge densities of the composite and isolated atom-graphene system, respectively. From the three-dimensional (3D) isosurfaces and 2D slices plotted for $\Delta \rho$, we find signatures of ionic and/or covalent bonds. Specifically, interaction with the alkali and alkali-earth-metal atoms such as $\mathrm{K}$ is ionic. There is no charge accumulation between the atom and graphene in this situation, in contrast to the transition-metal atoms such as $\mathrm{Fe}$ and $\mathrm{Cu}$, where covalent bonding features are identified. Moreover, the excess charge for $\mathrm{K} \mid \mathrm{G}$ is spatially delocalized, spreading on the upper surface of graphene, while for $\mathrm{Fe} \mid \mathrm{G}$ and $\mathrm{Cu} \mid \mathrm{G}$, it is localized on the carbon atoms next to the metal atom, also signaling a different bonding nature.

These arguments can be further validated by the electronic band structures, which were calculated by unfolding the results obtained for the supercells to the primitive cells $[31,32]$. The ion-graphene interaction can be characterized by the significance of Dirac points near the high-symmetry point 
(a)

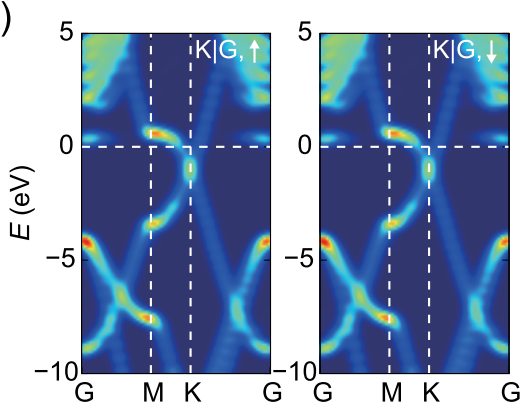

(b)

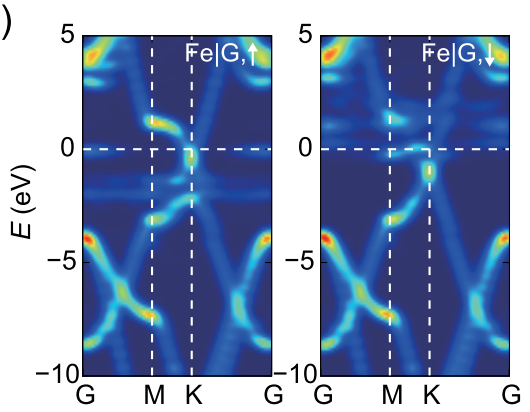

(c)

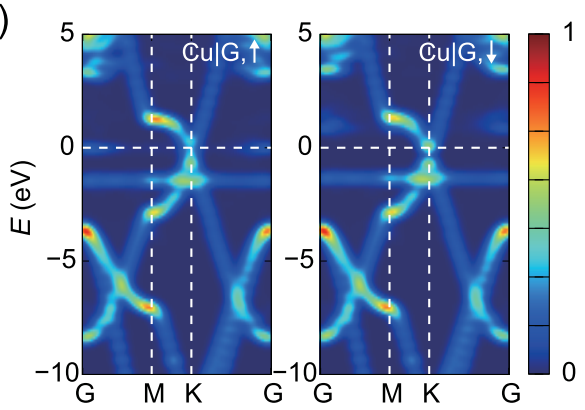

FIG. 2. Spin-resolved effective band structures (EBSs) of (a) $\mathrm{K} \mid \mathrm{G}$, (b) $\mathrm{Fe} \mid \mathrm{G}$, and (c) $\mathrm{Cu} \mid \mathrm{G}$ composites. The color bar indicates the Bloch spectral weight [31,32].

$\mathrm{K}$ in the effective band structure (EBS) [30,31,33]. Charge transfer between the ion and graphene shifts the Dirac point up or down with respect to the Fermi level (corresponding to $n$ - or $p$-type doping) [33]. Orbital projections show that electron accepting and donating is dominated by the $p_{z}$ orbital in graphene for all the ions under consideration, although charge transfers between the $s$ and $d$ orbitals are also identified in the transition-metal atoms [22]. For ionic interaction, the Dirac cone is distinct. In contrast, the covalent interaction perturbs the EBS by blurring the Dirac cone structures, as also indicated by electron localization between the adatom and graphene (Fig. 2) [22]. Analysis of density of states (DOS) and orbital projections identify that the perturbation is resulted from the coupling between $d$ electrons in the transition-metal atom and $p_{z}$ in graphene [22]. We also find that the significance of perturbation is positively correlated to the binding strength, although the characteristics of atomic orbitals are also important. Specifically, for $\mathrm{Fe}$ and $\mathrm{Ni}$ with $E_{\mathrm{b}}=0.854$ and $1.289 \mathrm{eV}$, the Dirac cone feature is destroyed, while for $\mathrm{Cu}$ and $\mathrm{Cr}$ with $E_{\mathrm{b}}=0.879$ and $0.374 \mathrm{eV}$, the modification is gentle (Fig. 2) [22].

Recent experimental studies on selective ion transport through graphene nanochannels show that the cation-anion and/or cation-cation selectivity can be achieved through mechanisms such as charge exclusion, size sieving, and cation- $\pi$ interaction as we discuss here [2-7]. To address the ion-graphene interaction from the ion mobility measured experimentally, the solvent effects are crucial [28,29]. Although the bare cation- $\pi$ interaction is notably stronger than van der Waals interactions, the strength of ion-water interaction in hydrated ions is on the same order [11,12]. The ion-wall interaction is thus expected to be weakened with the presence of solvation shells that separate the ion and graphene. By intercalating water molecules at the ion-graphene interface, we find that, surprisingly, the adsorption strength of the ionwater complex remains at the same order as that of the bare interface. This conclusion is preserved in calculations using several other functionals (Fig. S5), and is expected to hold for complexes with a larger hydration number, as validated by models with more than one solvation shell [22]. Water intercalation can modify the ionic degree of cations and the binding distance (Tables S3-S5) [22]. The binding strength $E_{\mathrm{b}}$ increases as the ion makes direct contact with graphene without water intercalated at the ion-graphene interface, as a result of the modified ionization of ions and graphene, but decreases for the complexes with water intercalation that screens out the interaction. The interaction between alkali-earth-metal ions and graphene remains ionic. For $\mathrm{Fe}$ and $\mathrm{Cu}$, the interaction changes from covalent to ionic as the ion-graphene distance increases (Figs. 3, S6, and S7) [22]. The Dirac cones in the (a)

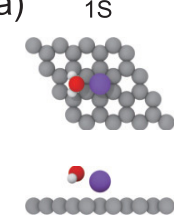

(e)

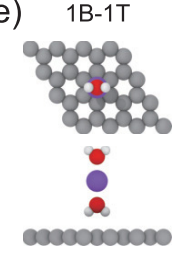

(b)

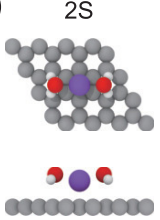

(f)

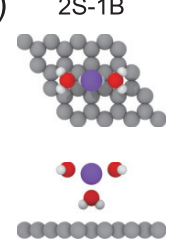

(c)

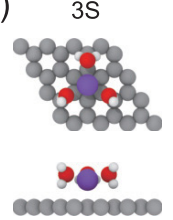

(g)

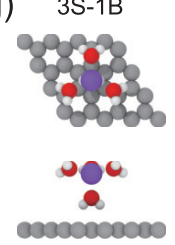

(d)

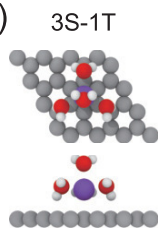

(h) $\quad 3 \mathrm{~S}-1 \mathrm{~B}-1 \mathrm{~T}$

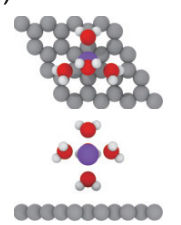

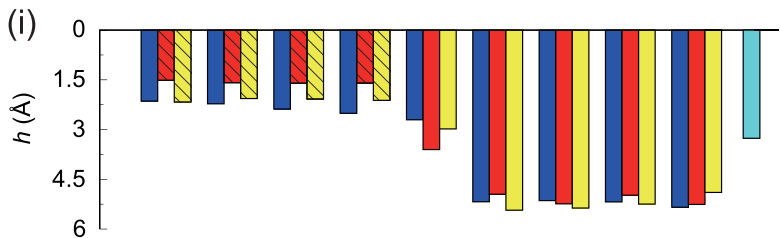

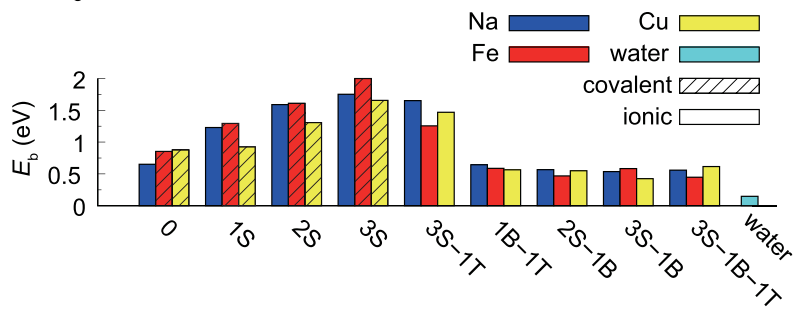

FIG. 3. [(a)-(h)] Optimized structures of Na/water complexes on graphene. S, T, and B denote the configurations with water molecules on the side, top, and bottom sites of $\mathrm{Na}$, respectively, in top and front views. The numerals before the letters indicate the number of water molecules. (i) Binding energies $\left(E_{\mathrm{b}}\right)$ calculated for $\mathrm{Na}, \mathrm{Fe}$, and $\mathrm{Cu}$ complexes. The dashed and undashed bars are the results for covalent and ionic interactions, respectively. The results for Na with more water molecules in the complex are shown in Table S3 and Fig. S8 [22]. 

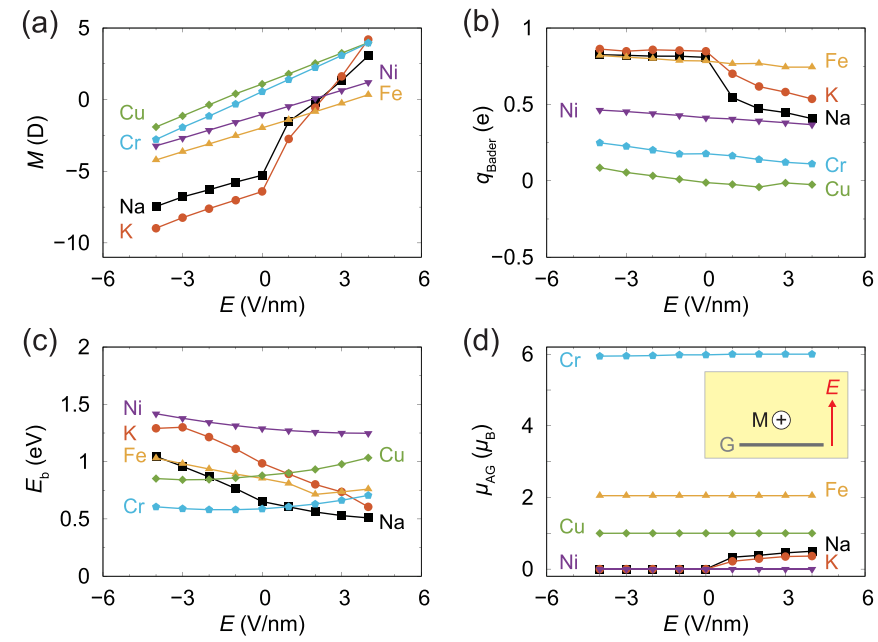

FIG. 4. (a) Dipole moments $(M)$, (b) Bader atomic charges $\left(q_{\text {Bader }}\right)$ [35], (c) binding energies $\left[E_{\mathrm{b}}=E_{\mathrm{M} \mid \mathrm{G}}(E)-E_{\mathrm{M}}(E)-\right.$ $\left.E_{\mathrm{G}}(0)\right]$, and (d) magnetic moments $\left(\mu_{\mathrm{M} \mid \mathrm{G}}\right)$ of the $\mathrm{M} \mid \mathrm{G}$ composites, plotted as a function of the strength $E$ of the EEF [illustrated in the inset of panel (d)]. Here $E_{\mathrm{M} \mid \mathrm{G}}(E)$ and $E_{\mathrm{M}}(E)$ are energies of the composite with a relaxed structure and an isolated ion under EEF, while $E_{\mathrm{G}}(0)$ is the energy of graphene with $E=0$.

EBSs of $\mathrm{Na}$ are distinct for all configurations. In contrast, for transition-metal ions such as $\mathrm{Fe}$ and $\mathrm{Cu}$, the Dirac cone feature is lost in the $2 S$ configuration, which, however, is recovered in the $2 S-1 B$ configurations as the $d$ electrons decouple with $p_{z}$ and the Dirac point is shifted (Figs. S6 and S7) [22].

The electrostatic nature of ion-graphene interaction suggests that the interaction can be engineered by applying the EEF $[13,34]$. Under an EEF in perpendicular to the basal plane of graphene with strength, $E$, ranging from -4 to $4 \mathrm{~V} / \mathrm{nm}$, the dipole moment of system $(M)$ changes continuously with the field strength $E$, indicating a modified charge-transfer behavior [Figs. 4(a) and 4(b)]. DFT calculation results show that the binding strength can be tuned by up to three times in the range of field strength explored here. For $\mathrm{Na}, \mathrm{K}, \mathrm{Fe}$, and $\mathrm{Ni}, E_{\mathrm{b}}$ increases with the field strength for $E<0$ and decreases for $E>0$, along with changes in the Bader atomic charge [Fig. 4(c)]. The difference in the field-strength dependence of $E_{\mathrm{b}}$ indicates that $\mathrm{EEF}$ provide an effective way to control the selective ion transport process as have been achieved in recent experiments [36]. The magnetic moment $\left(\mu_{\mathrm{M} \mid \mathrm{G}}\right)$ of the ion-graphene composite also change with $E$ for alkali and alkali-earth-metal ions, while for transition-metal ions it remains relatively intact [Fig. 4(d)]. Notably for $\mathrm{Na}$ and $\mathrm{K}, \mu_{\mathrm{M} \mid \mathrm{G}}$ increases from 0 to $\approx 0.5 \mu_{\mathrm{B}}$. This induced magnetic moment, although too weak to modify the ion-wall binding behaviors, implies potential applications of magnetoelectric effects in metal-free magnetic systems and single-atom magnetic storage [37,38]. The changes in the DOSs under EEF (Figs. S9-S11) [22] show that, for alkali composites of $\mathrm{K}$ and $\mathrm{Na}$, the $s$ orbitals are shifted, as a result of the charge transfer process. For transition-metal ions, the $d$ and $s$ orbitals of $\mathrm{Fe}$ and $\mathrm{Ni}$ are shifted, while for $\mathrm{Cu}$ and $\mathrm{Cr}$, the $s$ orbitals remains intact but the $d$ orbitals are shifted. This contrast may explain the distinct field-strength dependence of

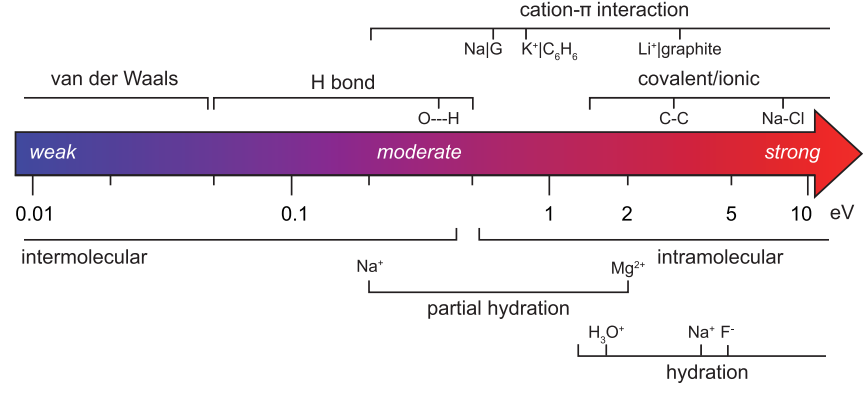

FIG. 5. Energy scales spanned by intermolecular (weak), intramolecular (strong), and cation- $\pi$ interactions $[11,13,14,28]$, the free energy of hydration and partial hydration of ions [2,39-41]. Specific values are annotated for the binding energy of $\mathrm{Na}$ on graphene (0.65 eV, Na|G using SCAN + rVV10) [22], $\mathrm{K}^{+}$on benzene $\left(0.83 \mathrm{eV}, \mathrm{K}^{+} \mid \mathrm{C}_{6} \mathrm{H}_{6}\right)$ [11], $\mathrm{Na}^{+}$on graphite $\left(2.35 \mathrm{eV}, \mathrm{Li}^{+} \mid\right.$graphite $)$ [14], the strength of hydrogen bond in bulk water $(0.24 \mathrm{eV})$ [42], strength of $\mathrm{C}-\mathrm{C}$ covalent bond $(3.57 \mathrm{eV})$ and $\mathrm{Na}-\mathrm{Cl}$ ionic bond $(8.16 \mathrm{eV})$ [43], free energy of partial hydration with the first hydration shell (1HS, $\approx 2 \mathrm{eV}$ for $\mathrm{Mg}^{2+}$ and $\approx 0.2 \mathrm{eV}$ for $\mathrm{Na}^{+}$) [2,40,41], and free energy of hydration for $\mathrm{H}_{3} \mathrm{O}^{+}(1.73 \mathrm{eV}), \mathrm{Na}^{+}(3.91 \mathrm{eV})$, and $\mathrm{F}^{-}(4.86 \mathrm{eV})$ [39].

$E_{\mathrm{b}}$ of $\mathrm{Cu}$ and $\mathrm{Cr}$ [Fig. 4(c)]. These findings suggest that, for carbon nanofluidics where the width of fluid channel is comparable with the range of ion-wall interaction, the selective ion transport or storage processes can be well controlled by external fields, which cannot be achieved with conventional knowledge in micro- and/or nanofluidics.

As the hydration free energy of ions $(\gtrsim 1.0 \mathrm{eV})$ is comparable to the strength of ion-graphene interaction [39], there is a competition between ion-graphene binding and ion solvation (Fig. 5) $[23,44]$. We identify that the partial dehydration free energy of the first hydration shell $\left(1 \mathrm{HS}, \approx 2 \mathrm{eV}\right.$ for $\mathrm{Mg}^{2+}$ and $\approx 0.2 \mathrm{eV}$ for $\left.\mathrm{Na}^{+}\right)[2,40,41]$ is comparable to the strength of ion-graphene interaction and that of hydrogen bonds in water (0.24 eV [42]). Previous studies suggest that weakly hydrated ions such as $\mathrm{Na}^{+}$and $\mathrm{K}^{+}$can make direct contact with the graphene surface, but not for highly hydrated ions such as $\mathrm{Ca}^{2+}$ and $\mathrm{Mg}^{2+}[23,44]$. In nanochannels, perturbation on HSs from complexity in the chemical environment $[15,23]$, modification of water-structuring effects (structuremaking, structure-breaking) [45], paired correlation of ions [36], and potential of external fields are coupled to the ionwall interaction. The configurations of hydrated ions in the graphene nanochannels are thus not easy to predict. Recent advances in experimental techniques may advance our understandings beyond the theoretical arguments. For example, experiments using scanning tunneling microscopy (STM) and atomic force microscopy (AFM) demonstrated the feasibility of exploring HSs near a surface [46,47]. One can also use x-ray absorption spectroscopy (XAS), second-harmonic generation (SHG) microscopy, and other tools along with molecular dynamics (MD) simulations to probe the HSs indirectly [48,49]. Further experiments and simulations with more detailed information on the ion-wall interaction are needed to clarify these points. Since the specific, relatively strong, and robust interaction between ions and graphene stems from electrostatic interaction and/or covalent bonding 
(compared to the van der Waals interaction), the polarization in ion-graphene composites requires an improved treatment far beyond the simple pair-form potentials such as the L-J and Morse function. Moreover, the delocalization of electrons transferred to and from graphene makes it challenging to describe the ion-graphene interaction [22]. Our work thus offers key insights to develop accurate force fields for classical MD simulations of ion dynamics confined in graphene channels where the ion-wall interaction becomes essential, which can be implemented by, for example, using the deep-learning- based representation to address the accuracy versus efficiency dilemma [50].

This study was supported by the MIT-China seed fund, the National Natural Science Foundation of China through Grants No. 11472150, No. 11825203, and No. 11921002, and the National Key Basic Research Program of China through Grant No. 2015CB351900. The computation was performed on the Explorer 100 cluster system of Tsinghua National Laboratory for Information Science and Technology.
[1] L. Wang, M. S. Boutilier, P. R. Kidambi, D. Jang, N. G. Hadjiconstantinou, and R. Karnik, Nat. Nanotechnol. 12, 509 (2017).

[2] J. Abraham, K. S. Vasu, C. D. Williams, K. Gopinadhan, Y. Su, C. T. Cherian, J. Dix, E. Prestat, S. J. Haigh, I. V. Grigorieva et al., Nat. Nanotechnol. 12, 546 (2017).

[3] R. Joshi, P. Carbone, F. C. Wang, V. G. Kravets, Y. Su, I. V. Grigorieva, H. Wu, A. K. Geim, and R. R. Nair, Science 343, 752 (2014)

[4] S. Hong, C. Constans, M. V. Surmani Martins, Y. C. Seow, J. A. Guevara Carrió, and S. Garaj, Nano Lett. 17, 728 (2017).

[5] P. Sun, M. Zhu, K. Wang, M. Zhong, J. Wei, D. Wu, Z. Xu, and H. Zhu, ACS Nano 7, 428 (2012).

[6] P. Sun, F. Zheng, M. Zhu, Z. Song, K. Wang, M. Zhong, D. Wu, R. B. Little, Z. Xu, and H. Zhu, ACS Nano 8, 850 (2014).

[7] P. Sun, K. Wang, and H. Zhu, Adv. Mater. 28, 2287 (2016).

[8] E. D. Glendening, D. Feller, and M. A. Thompson, J. Am. Chem. Soc. 116, 10657 (1994).

[9] E. D. Glendening and D. Feller, J. Am. Chem. Soc. 118, 6052 (1996).

[10] E. T. Acar, S. F. Buchsbaum, C. Combs, F. Fornasiero, and Z. S. Siwy, Sci. Adv. 5, eaav2568 (2019).

[11] J. C. Ma and D. A. Dougherty, Chem. Rev. 97, 1303 (1997).

[12] D. A. Dougherty, Science 271, 163 (1996).

[13] M. Novák, C. Foroutan-Nejad, and R. Marek, J. Chem. Theory Comput. 12, 3788 (2016).

[14] G. Shi, J. Liu, C. Wang, B. Song, Y. Tu, J. Hu, and H. Fang, Sci. Rep. 3, 3436 (2013).

[15] L. Chen, G. Shi, J. Shen, B. Peng, B. Zhang, Y. Wang, F. Bian, J. Wang, D. Li, Z. Qian et al., Nature (London) 550, 380 (2017).

[16] J. P. Gallivan and D. A. Dougherty, Proc. Natl. Acad. Sci. U.S.A. 96, 9459 (1999).

[17] K. Fujisawa, C. Beuchat, M. Humbert-Droz, A. Wilson, T. A. Wesolowski, J. Mareda, N. Sakai, and S. Matile, Angew. Chem. Int. Ed. 53, 11266 (2014).

[18] B. Hille, Ion Channels of Excitable Membranes (Sinauer Associates Inc., Sunderland, MA, 2001), Vol. 507.

[19] S. Mecozzi, A. P. West, and D. A. Dougherty, Proc. Natl. Acad. Sci. U.S.A. 93, 10566 (1996).

[20] J. Sun, A. Ruzsinszky, and J. P. Perdew, Phys. Rev. Lett. 115, 036402 (2015).

[21] H. Peng, Z.-H. Yang, J. P. Perdew, and J. Sun, Phys. Rev. X 6, 041005 (2016).
[22] See Supplemental Material at http://link.aps.org/supplemental/ 10.1103/PhysRevResearch.2.042034 for more detailed information about the binding energies calculated from 12-6 L-J potential functions, the effects of exchange-correlation functionals and $\mathrm{vdW}$ corrections, the effects of lateral cell sizes and vacuum layer thickness, the comparison with van der Waals interactions, and supplementary tables, figures.

[23] K. Zhou and Z. Xu, ACS Appl. Mater. Interfaces 10, 27801 (2018).

[24] G. Kresse and J. Furthmüller, Phys. Rev. B 54, 11169 (1996).

[25] W. Zhang, J. Lin, W. Xu, H. Fu, and G. Yang, Tsinghua Sci. Technol. 22, 675 (2017).

[26] G. Makov and M. C. Payne, Phys. Rev. B 51, 4014 (1995).

[27] P. E. Blöchl, Phys. Rev. B 50, 17953 (1994).

[28] S. Meng, D. V. Chakarov, B. Kasemo, and S. Gao, J. Chem. Phys. 121, 12572 (2004).

[29] S. Meng and S. Gao, J. Chem. Phys. 125, 014708 (2006).

[30] K. T. Chan, J. B. Neaton, and M. L. Cohen, Phys. Rev. B 77, 235430 (2008).

[31] P. V. C. Medeiros, S. Stafström, and J. Björk, Phys. Rev. B 89, 041407(R) (2014).

[32] O. Rubel, A. Bokhanchuk, S. J. Ahmed, and E. Assmann, Phys. Rev. B 90, 115202 (2014).

[33] G. Giovannetti, P. A. Khomyakov, G. Brocks, V. M. Karpan, J. van den Brink, and P. J. Kelly, Phys. Rev. Lett. 101, 026803 (2008).

[34] M. Novák, C. Foroutan-Nejad, and R. Marek, Phys. Chem. Chem. Phys. 18, 30754 (2016).

[35] R. F. Bader, Acc. Chem. Res. 18, 9 (1985).

[36] C. Cheng, G. Jiang, G. P. Simon, J. Z. Liu, and D. Li, Nat. Nanotechnol. 13, 685 (2018).

[37] Z. Zhang, C. Chen, and W. Guo, Phys. Rev. Lett. 103, 187204 (2009).

[38] F. D. Natterer, K. Yang, W. Paul, P. Willke, T. Choi, T. Greber, A. J. Heinrich, and C. P. Lutz, Nature (London) 543, 226 (2017).

[39] Y. Marcus, Ions in Solution and Their Solvation (John Wiley \& Sons, New York, 2015).

[40] K. Zhou and Z. Xu, J. Phys. Chem. C 124, 4890 (2020).

[41] Y. Yu, J. Fan, J. Xia, Y. Zhu, H. Wu, and F. Wang, Nanoscale 11, 8449 (2019).

[42] S. Suresh and V. Naik, J. Chem. Phys. 113, 9727 (2000).

[43] J. E. Huheey, E. A. Keiter, and R. L. Keiter, Inorganic Chemistry: Principles of Structure and Reactivity, 4th ed. (HarperCollins, New York, 1993). 
[44] C. D. Williams, J. Dix, A. Troisi, and P. Carbone, J. Phys. Chem. Lett. 8, 703 (2017).

[45] G. Stirnemann, E. Wernersson, P. Jungwirth, and D. Laage, J. Am. Chem. Soc. 135, 11824 (2013).

[46] M. Ricci, P. Spijker, and K. Voïtchovsky, Nat. Commun. 5, 4400 (2014).

[47] J. Peng, D. Cao, Z. He, J. Guo, P. Hapala, R. Ma, B. Cheng, J. Chen, W. J. Xie, X.-Z. Li et al., Nature (London) 557, 701 (2018).
[48] J.-J. Velasco-Velez, C. H. Wu, T. A. Pascal, L. F. Wan, J. Guo, D. Prendergast, and M. Salmeron, Science 346, 831 (2014).

[49] D. L. McCaffrey, S. C. Nguyen, S. J. Cox, H. Weller, A. P. Alivisatos, P. L. Geissler, and R. J. Saykally, Proc. Natl. Acad. Sci. U.S.A. 114, 13369 (2017).

[50] H. Wang, L. Zhang, J. Han, and W. E., Comput. Phys. Commun. 228, 178 (2018). 